\title{
Advantaged Outcomes of Preemptive Living Donor Kidney Transplantation and the Effect of Bias
}

\author{
Gadelkareem RA ${ }^{1}$, Zarzour MA ${ }^{1}$, Khalil $\mathbf{M}^{1}$, Azoz NM ${ }^{2}$, Reda A, Abdelgawad AM$^{1}$, Mohammed N $^{1,3}$ and Hammouda \\ HM $^{1}$
}

${ }^{1}$ Assiut Urology and Nephrology Hospital, Faculty of Medicine, Assiut University, Assiut, Egypt

${ }^{2}$ Department of Internal Medicine-Nephrology Unit, Faculty of Medicine, Assiut University, Assiut, Egypt

${ }^{3}$ Urology Department, Martin-Luther University, Halle, Germany

*Corresponding author: Gadelkareem RA, Assiut Urology and Nephrology Hospital, Assiut University, Assiut, Egypt. 71515; dr.rabeagad@yahoo.com

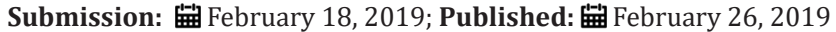

\begin{abstract}
Preemptive living donor kidney transplantation is the optimal treatment of end-stage renal disease with advantaged outcomes when it is compared to transplantation after starting chronic dialysis. However, these advantages are concerned to be affected by the lead-time and patients' selection bias effects. We classified the potential biasing factors into intrinsic and extrinsic factors to the process of kidney transplantation and presented a different perspective in the effect of bias. We considered these factors as mere characteristics in the process of preemptive living donor kidney transplantation which should be provided in accurate timing. Therefore, preemptive and non-preemptive kidney transplantations represent a single line of treatment which is implemented at different timings along the course of end-stage renal disease rather than two parallel lines. Hence, the perspective of lead-time bias could be ameliorated.
\end{abstract}

Keywords: End-stage renal disease; Kidney transplantation; Lead-time bias; Living donors; Preemptive kidney transplantation

Abbreviations: CKD: Chronic Kidney Disease; ESRD: End-Stage Renal Disease; KT: Kidney Transplantation; LDKT: Living Donor Kidney Transplantation; PKT: Preemptive Kidney Transplantation

\section{Introduction}

Preemptive Kidney Transplantation (PKT) is defined as Kidney Transplantation (KT) before starting chronic dialysis [1,2]. Chances of receiving PKT are more likely with the availability of living kidney donors, but only one third of the Living Donor Kidney Transplantations (LDKT) currently receives PKT [1-3]. These shortcomings in the progress of PKT could be principally attributed to delayed referral of patients to transplantation centers and a few unfavourable concerns about PKT [3-5]. PKT, generally, or preemptive living donor kidney transplantation, specifically, provides better outcomes among both the paediatric and adult populations $[1,4,6]$. However, these advantaged outcomes in all the reported studies have been concerned to be promoted by bias in many aspects such as transplantation with higher residual native renal functions, relatively healthier recipients, and higher socioeconomic conditions of the patients who access to PKT $[4,5,7]$. Our opinion in this notion is different and represents a practical rather than a statistical perspective.

\section{Opinion}

The presence of bias in patients' selection on studying the outcomes of PKT has been frequently raised in the literature. It has been reported that recipients of PKT have better medical and socio-economic conditions which may contribute to the advantaged outcomes [4,5]. In most instances, the researchers have adopted such a perspective in which PKT and non-PKT are considered as two modalities of treatment of End-Stage Renal Disease (ESRD), hence the lead-time bias has been proposed [4,7]. However, they are and should be considered as one modality of treatment which is KT surgery and it could be provided in different sequential stages or periods of ESRD along the scale of time. According to the view which supports the presence of bias, the proposed bias has been attributed to different medical and socio-economic factors $[4,7]$. We can classify these factors into, either, intrinsic or extrinsic factors to the process of management of ESRD to represent our perspective.

\section{Intrinsic factors in the process of management of ESRD}

Firstly, early referral to transplantation centers with better general conditions and availability of living donors are the two main factors which facilitate the access to PKT. Early presentation to transplantation center in the course of ESRD enhances LDKT. According to how much rapidly a potential recipient matches with a suitable potential donor, his chances in PKT become increasingly available $[4,8]$. So, enrolling of the potential recipients of PKT in randomized studies seems to be unlikely [4,5]. If randomization is planned, many of these cases will bypass the chances of better management. 
PKT is a timing strategy for treatment of the late stages of Chronic Kidney Disease (CKD) [7,9]. This strategy is the accurate offering and implementation of KT, while the patient is traversing the trajectory of ESRD. This means that the method of treatment by KT is a surgical procedure which is technically not different in PKT and non-PKT, but the time of implementation of this technique is the variable element. So, the patient gains more morbidities, while time is going on and the outcomes become less favourable. To simplify this notion, we may resort to simulation of the progress of ESRD to a prominent example of staged diseases in medicine such as malignancy. For example, renal cell carcinoma is a wellknown pathology that has been staged into four definite stages and radical nephrectomy is its gold standard treatment, where it increasingly provides better outcomes in the early stages of disease [10]. If comparisons are done between the outcomes of radical nephrectomy in early and late stages, the outcomes of these comparisons will be definitively biased towards the early stages. According to the duration of dialysis, ESRD patients who could be treated by KT have been categorized into multiple consecutive categories. Categorization of timing is either in days or months such as patients who had KT prior to dialysis, after short duration of dialysis (6-12 months), and after long duration of dialysis $[9,11]$. Similar to the above example of malignancy, outcomes of kidney transplantation should be definitively better in the early stages of ESRD rather than the late stages. Hence, the proposed lead-time bias is a mere character of the process of PKT and it will not be removed under the design of early KT or PKT.

This explanation makes the trials to put PKT and non-PKT approaches in parallel comparisons to avoid bias in selection is impossible. Similar to malignancy, ESRD is furtherly categorized or staged and its treatment by KT is ultimately better in the early stages. Despite the debate about the optimal values at which PKT should be offered, PKT is limited mostly to patients with glomerular filtration rates $<15 \mathrm{ml} / \mathrm{min} / 1.73 \mathrm{~m}^{2}$ and is not justified for higher levels of renal functions $[4,9,12]$. On the other hand, the absolute early PKT in the course of CKD is not a logic approach in practice, where it will be confronted to the major risks of early exposure to surgery and immunosuppressive therapy. However, these risks make this exposure justified only when they are outweighed by the benefits of PKT $[4,9]$. Hence, the adjusted timing of PKT has been configured [7]. Unless these limitations are present, the recipients of early PKT should be imaginarily healthier at the early stages of CKD and should have more advantageous outcomes. However, this proposed scenario could not be proofed practically [9]. Therefore, it is a matter of accurate timing in the track of the CKD and ESRD rather than a matter of different modalities of treatment.

Secondly, LDKT provides better outcomes than deceased donor KT. It has been found that LDKT provides better chances to PKT. The combination of PKT and LDKT provides a synergistic effect to the process of KT $[4,8]$. Like the early referral to KT centers, availability of the living donors is a mere element in the LDKT. In-center and outreach education programmes about the advantages of LDKT increases the early wait listing and PKT [8].

\section{Extrinsic factors from the surroundings}

In PKT, socio-economic conditions are represented by the higher education levels, more wealthy states, higher rates of employment, more being married rather than single, and having private insurance coverage $[4,5,13]$. Ultimately, private insurance coverage and higher education levels could be considered as surrogates for other characteristics such as higher rates of employment and therefore more wealthy states. Moreover, all the higher socioeconomic conditions could be considered as surrogates for the intrinsic factors of the whole process and early access to KT [5,14]. In turn, this will support the concept of the PKT process as it has been explained formerly. Socio-economic conditions could be improved by incorporation into national financial coverage and health education programmes [5,14].

In conclusion, our perspective is that if the advantaged outcomes of PKT are statistically related to these proposed biasing conditions, they should be considered as promotors rather than confounders to PKT programmes. This is explained by that PKT and no-PKT are not parallel approaches, but they are one approach that could be implemented in consecutive stages of ESRD. PKT is considered as an early step in management of ESRD which should be viewed in such a manner like the other sequentially staged diseases such as malignancy. Accordingly, the proposed lead-time bias is a mere finding in the process of PKT strategy. Removal of lead-time bias means amelioration of the whole PKT process and its advantages.

\section{References}

1. Jay CL, Dean PG, Helmick RA, Stegall MD (2016) Reassessing preemptive kidney transplantation in the United States: Are we making progress? Transplantation 100(5): 1120-1127.

2. Chopra B, Sureshkumar KK (2018) Kidney transplantation in older recipients: Pre-emptive high KDPI kidney vs lower KDPI kidney after varying dialysis vintage. World J Transplant 8(4): 102-109.

3. Helmick RA, Jay CL, Price BA, Dean PG, Stegall MD (2018) Identifying barriers to pre-emptive kidney transplantation in a living donor transplant cohort. Transplantation Direct 4(4): e356.

4. Abramowicz D, Hazzan M, Maggiore U, Peruzzi L, Cochat P, et al. (2015) Does pre-emptive transplantation versus post start of dialysis transplantation with a kidney from a living donor improve outcomes after transplantation? A systematic literature review and position statement by the Descartes working group and ERBP. Nephrol Dial Transplant 31(5): 691-697.

5. Kasiske BL, Snyder JJ, Matas AJ, Ellison MD, Gill JS, et al. (2002) Preemptive kidney transplantation: The advantage and the advantaged. J Am Soc Nephrol 13(5): 1358-1364.

6. Amaral S, Sayed BA, Kutner N, Patzer RE (2016) Pre-emptive kidney transplantation is associated with survival benefits among paediatric patients with end-stage renal disease. Kidney Int 90(5): 1100-1108.

7. Friedewald JJ, Reese PP (2012) The kidney-first initiative: what is the current status of pre-emptive transplantation? Adv Chronic Kidney Dis 19(4): 252-256.

8. Waterman AD, Morgievich M, Cohen DJ, Butt Z, Chakkera HA, et al. (2015) Living donor kidney transplantation: Improving education outside of transplant centres about live donor transplantation-Recommendations from a consensus conference. Clin J Am Soc Nephrol 10(9): 1659-1669. 
9. Akkina SK, Connaire JJ, Snyder JJ, Matas AJ, Kasiske BL (2008) Earlier is not necessarily better in pre-emptive kidney transplantation. Am J Transplant 8(10): 2071-2076.

10. Krabbe LM, Bagrodia A, Margulis V, Wood CG (2014) Surgical management of renal cell carcinoma. Semin Intervent Radiol 31(1): 27 32.

11. Goldfarb RA, Hurdle JF, Scandling J, Wang Z, Baired B, et al. (2005) Duration of end-stage renal disease and kidney transplant outcome. Nephrol Dial Transplant 20(1): 167-175.

12. Fissell RB, Srinivas T, Fatica R, Nally J, Navaneethan S, et al. (2012) Preemptive renal transplant candidate survival, access to care, and renal function at listing. Nephrol Dial Transplant 27(8): 3321-3329.
13. Khattak MW, Sandhu GS, Woodward R, Stoff JS, Goldfarb RAS (2010) Association of marital status with access to renal transplantation. Am J Transplant 10(12): 2624-2631.

14. Harhay MN, Kenna RM, Boyle SM, Ranganna K, Mizrahi LL, et al. (2018) Association between medicaid expansion under the affordable care act and pre-emptive listings for kidney transplantation. Clin J Am Soc Nephrol 13(7): 1069-1078.
Creative Commons Attribution 4.0 International License

For possible submissions Click Here

\begin{tabular}{|c|c|c|}
\hline ETUN & 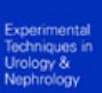 & $\begin{array}{c}\text { Experimental Techniques in Urology \& Nephrology } \\
\text { Benefits of Publishing with us }\end{array}$ \\
\hline & & $\begin{array}{l}\text { - High-level peer review and editorial services } \\
\text { - Freely accessible online immediately upon publication } \\
\text { - Authors retain the copyright to their work } \\
\text { - Licensing it under a Creative Commons license } \\
\text { - Visibility through different online platforms }\end{array}$ \\
\hline
\end{tabular}

